Patients (or Participants) 192 elite female netball players from all 16 contesting teams at the NWC (average squad size 12 players).

Interventions (or Assessment of Risk Factors) Medical staff of each team recorded all illness (1440 player days) and training/ match days lost during the 10-day tournament.

Main Outcome Measurements Incidence rates (IR) (per 1000 player-days; 95\% CI) and severity (\% time-loss $\geq 1$ day) of all illness.

Results In total 11 illnesses were recorded. The overall crude IR of illness was $7.6(3.1-12.2)$. Organ systems most affected was the respiratory- (27\%), followed by gastrointestinal$(18 \%)$ and urogenital tract $(18 \%)$. More common illness, by specific diagnosis, was acute upper respiratory infection (18\%), non-infective gastroenteritis (18\%) and dysmenorrhea (18\%). Cause of illness was similar for non-infective $(5.6 ; 1.7-9.4)$ and infective $(2.1 ; 0.3-4.4)$ illness $(p=0.227)$. Time-loss of $\geq 1$ day was reported for $9 \%$ of all illness. Illness by player position was common in goal keepers (27\%), goal defenders (27\%), followed by goal shooters (18\%). Illness was reported on a match day in $81 \%$ cases (match day with a training session $=45 \%$; match day without training $=36 \%$ ).

Conclusions There is a moderate incidence of illness during the 2019 NWC, but we note that $>80 \%$ of players only report illness on match days. We suggest that players be educated to report illness early (at onset of first symptoms), so that treatment can commence before match day. We recommend implementing illness prevention programs prior to and at future international netball tournaments.

\section{THE RELIABILITY AND ASSOCIATION OF THE REPEATED COPENHAGEN ADDUCTION EXERCISE TO GAELIC FOOTBALL PLAYERS WITH A HISTORY OF GROIN INJURY: A PILOT STUDY}

Rachel Tierney, Ahmad Salma, Ulrik McCarthy Persson. University College Dublin, Dublin, Ireland

\subsection{6/bjsports-2021-IOC.355}

Background The Copenhagen adduction exercise (CAE) can significantly reduce the risk of developing Groin injuries (GIs) and may be of clinical utility in being used as a test to assess hip-adductor muscle function.

Objective To examine the relationship between hip-adductor muscle function using a modified, repeated version of the CAE with the adductor squeeze test and the function, sport and recreation subscale of the Copenhagen hip and groin outcome score (HAGOS) in male Gaelic football players (GFP) with a history of GIs. A secondary aim was to assess the inter-rater reliability of the CAE.

Design Two stage pilot study. Stage one assessed the inter-rater reliability of the CAE in active male university students. Stage 2 examined the relationship between hip-adductor muscle function using the $\mathrm{CAE}$ with the adductor squeeze test and level of function in male GFP with and without a history of Gis.

Setting University clinical skills laboratory and Gaelic football clubs.

Participants Recruitment on a volunteer basis. Thirteen physically active males over 18 with no history of GIs participated in stage one. Sixty-two males (previous GIs $n=30$, no previous GIs $\mathrm{n}=32$ ) over 18 years, currently playing Gaelic participated in stage two. To be included into the 'injured' group, participants needed to have a history of GIs diagnosed by a physiotherapist which resulted in complete absence from training for at least two weeks. Participants were excluded if they had an acute injury ( $<2$ weeks) or were unable to participate in training sessions.

Main Outcome Measurements Maximum number of repetitions achieved during the CAE, adductor squeeze test and the HAGOS function, sport and recreation subscale.

Results The CAE test demonstrated good inter-rater reliability (ICC: 0.86, 95\% CI: 0.61-0.96, p<0.001). Injured and uninjured Gaelic footballers did not differ in CAE or adductor squeeze scores $(\mathrm{p}>0.05)$. Injured players did not differ in CAE scores between their injured and uninjured leg $(p=0.127)$. There was a significant correlation between the CAE score on the uninjured leg and the HAGOS $(\rho=0.405$; $\mathrm{p}=0.026)$ and adductor squeeze $(\rho=0.458 ; \mathrm{p}=0.011)$.

Conclusions The CAE has good inter-rater reliability. The repeated CAE test was unable to differentiate between injured and uninjured Gaelic football players or between injured and uninjured legs.

\section{ABSTRACT WITHDRAWN}

\section{CAN A HEAT-AND-MOISTURE EXCHANGER ATTENUATE INFLAMMATORY RESPONSES TO EXERCISE IN SUB- ZERO CONDITIONS?}

${ }^{1}$ Helen G Hanstock, ${ }^{1}$ Alasdair S Tutt, ${ }^{2}$ Hampus Persson, ${ }^{1}$ Erik P Andersson, ${ }^{3}$ Mats Ainegren, ${ }^{2}$ Nikolai Stenfors. 'Swedish Winter Sports Research Centre, Department of Health Sciences, Mid Sweden University, Östersund, Sweden; ${ }^{2}$ Unit of Medicine, Department of Public Health and Clinical Medicine, Umeå University, Umeå, Sweden; ${ }^{3}$ Sports Tech Research Centre, Department of Quality Management and Mechanical Engineering, Mid Sweden University, Östersund, Sweden

\subsection{6/bjsports-2021-IOC.356}

Background Heavy endurance training in sub-zero environments increases risk of exercise-induced asthma. Heat-andmoisture exchangers (HME) can prevent exercise-induced bronchoconstriction but it is not known whether they protect against inflammatory responses to exercise in healthy individuals.

Objective To investigate whether use of an HME during exercise in a sub-zero environment affects post-exercise inflammatory responses.

Design Investigator-blind randomised crossover trial.

Setting Environmental chamber at $-15^{\circ} \mathrm{C}$.

Participants 23 healthy, trained participants aged 18-53 (15 male, 8 female, $\mathrm{VO}_{2 \text { peak }} 57 \pm 6$ and $50 \pm 4 \mathrm{~mL} / \mathrm{kg} / \mathrm{min}$; mean $\pm \mathrm{SD})$.

Interventions Two experimental trials with and without HME, consisting of 30-min moderate-intensity running followed by a 4-min maximal running time-trial. Plasma samples were obtained pre- and 1h-post-exercise and analysed for a panel of 10 cytokines using a multiplex immunoassay.

Main Outcome Measurements Plasma cytokine concentrations (GM-CSF，IL-1 $\beta$, IL-4，IL-5，IL-6，IL-8，IL-10，IL-13，IL-17E/ 25, TNF- $\alpha$ ). Data were log-transformed then analysed using two-way repeated-measures ANOVA; one participant was an extreme outlier and excluded. 
Results Five cytokines (GM-CSF, IL-1 $\beta$, IL-4, IL-13, IL-17E/ $25)$ returned $<20 \%$ concentrations within detection limits and were excluded from further analysis. The other cytokines returned $>85 \%$ samples in range. IL-6, IL-8 and IL-10 increased after exercise (IL-6: $F=36, \mathrm{p}<0.0001$; IL-8: $F=39$, $\mathrm{p}<0.0001$; IL-10: $F(1,21)=8.9, \mathrm{p}=0.0072)$. There was a trend towards a greater post-exercise increase in IL-10 with HME (HME: median 0.062 (range $-0.203-1.053$ ) pg/mL; no-HME: $0.047(-0.079-0.50) \mathrm{pg} / \mathrm{mL} ; F=3.0, \mathrm{p}=0.096)$. There were no significant interactions for other cytokines.

Conclusions Use of an HME during exercise in a sub-zero environment did not affect systemic pro- and anti-inflammatory cytokine responses to exercise. Local inflammatory markers in the lungs may be relevant to investigate in future studies.

\section{A COMPARISON OF THE PREVALENCE AND SEVERITY OF HEALTH PROBLEMS IN NATIONAL TEAM CROSS- COUNTRY SKIERS OVER A COMPETITIVE SEASON}

${ }^{1}$ Øyvind Karlsson, ${ }^{2}$ Magnus Danemar, 'Marko S Laaksonen, 'Kerry McGawley. 'Swedish Winter Sports Research Centre, Department of Health Sciences, Mid Sweden University, Östersund, Sweden; 'Department of Physiology and Pharmacology, Karolinska Institutet, Stockholm, Sweden

\subsection{6/bjsports-2021-IOC.357}

Background A limited number of long-term prospective studies have investigated health problems in competitive cross-country skiers. Moreover, limited information exists regarding differences in the prevalence and severity of health problems between senior and development female and male cross-country skiers. Objective To compare the prevalence and severity of health problems between senior and development female and male national team cross-country skiers.

Design Comparative, prospective, observational cohort study.

Setting Senior and development national cross-country ski teams.

Participants Eighteen (9 women, 9 men) senior and 22 (9 women, 13 men) development national team skiers.

Intervention Skiers self-reported their health problems weekly for 17 weeks throughout the 2019/2020 competitive crosscountry ski season using the Oslo Sports Trauma Research Center Questionnaire on Health Problems (OSTRC-H2).

Main Outcome Measurements We calculated weekly prevalence and severity measures for illnesses, acute and overuse injuries, substantial health problems (i.e., health problems leading to moderate or severe reductions in training volume or sports performance, or a complete inability to participate) and all health problems combined.

Results Weekly response rate to the OSTRC-H2 was 90\% (SD $7 \%)$. Average weekly prevalence of substantial $(\sim 12 \%)$ and all $(\sim 25 \%)$ health problems were similar between senior and development team skiers. However, illness prevalence was lower in senior vs. development skiers $(8 \%, 95 \%$ CI $[3 \%$, $13 \%]$ vs. $13 \%, 95 \%$ CI $[9 \%, 17 \%]$, respectively; $p=.026)$ and injuries were higher $(12 \%, 95 \%$ CI $[9 \%, 15 \%]$ vs. $5 \%$, $95 \%$ CI $[3 \%, 7 \%] ; \mathrm{p}<.001)$. There were no differences in severity measures (all $\mathrm{p}>.05$ ).

Conclusions Health problems were relatively common among the skiers, with one quarter affected by at least one problem at any given time. While the prevalence of all health problems was similar, illnesses were less prevalent in the senior compared to the development team skiers and injuries were more prevalent.

\section{THE PREVALENCE AND BURDEN OF HEALTH PROBLEMS IN MALE SENIOR ICE HOCKEY: A PROSPECTIVE STUDY IN THE NORWEGIAN PROFESSIONAL LEAGUE}

${ }^{1,2}$ Anine Nordstrøm, ${ }^{1}$ Roald Bahr, ${ }^{2}$ Ove Talsnes, ${ }^{1}$ Benjamin Clarsen. ${ }^{1}$ Department of Sports Medicine, Oslo Sports Trauma Research Center, Norwegian School of Sports Sciences, Oslo, Norway; ${ }^{2}$ Innlandet Hospital Trust, Elverum, Norway

\section{$10.1136 /$ bjsports-2021-IOC.358}

Background As previous epidemiological studies in elite ice hockey have focused on acute time-loss injuries, little is known about the burden of overuse injuries and illnesses in ice hockey.

Objective The aim of this study was to report the prevalence and burden of all health problems in male senior elite ice hockey during one competitive season.

Design Prospective cohort study.

Setting The 2017/2018 competitive season of the GET league (the premier professional league) in Norway.

Patients (or Participants) We included nine of the ten teams in the GET league (the premier professional league) in Norway. In the nine teams participating, five players declined to participate, six players did not report and one player dropped out. A total of 225 players finished the study.

Interventions (or Assessment of Risk Factors) Players reported all health problems; acute injuries, overuse injuries and illnesses, for 31 weeks using Oslo Sports Trauma Research Center Questionnaire on Health Problems.

Main Outcome Measurements Prevalence and burden of acute injuries, overuse injuries and illnesses.

Results At any given time, 40\% (95\% CI 37\% to $43 \%$ ) reported symptoms from injury or illness, and 20\% $95 \% \mathrm{CI}$ $19 \%$ to $22 \%$ ) experienced health problems with a substantial negative impact on training and performance. Acute injuries represented the greatest incidence, prevalence and burden. The most burdensome acute injuries were to the head/face, shoulder, knee and ankle. The most burdensome overuse injury locations were knee, lumbar spine and hip/groin.

Conclusions This registration did capture a greater burden from overuse injuries than traditional injury registration, but acute injuries did represent the major problem.

\section{PROMOTION FOR JAPAN SKIING SAFETY}

Yukio Urabe, Kazuki Fukui, Junpei Sasadai, Makoto Komiya, Masanori Morikawa, Noriaki Maeda. Graduate School of Biomedical and Health Sciences, Hiroshima University, Hiroshima, Japan

\subsection{6/bjsports-2021-IOC.359}

Background Niseko, which is located north of Japan, becomes a famous ski resort for the best powder snow called as 'JAPOW' or 'NISEPOW'. As the number of backcountry skiing's popularity increased, accidents related skiing also did in Niseko.

Objective The purpose was to show the effort for the safety of skiers in Niseko and how to prevent and decrease the number of ski accidents in Japan.

Design Cross-sectional study.

Setting Field of Niseko skiing resort. 